\title{
Experimental Measurement of Self-Diffusion in a Strongly Coupled Plasma
}

\author{
T. S. Strickler, ${ }^{1}$ T. K. Langin, ${ }^{1}$ P. McQuillen, ${ }^{1}$ J. Daligault, ${ }^{2}$ and T. C. Killian ${ }^{1}$ \\ ${ }^{1}$ Department of Physics and Astronomy, Rice University, Houston, Texas 77005, USA \\ ${ }^{2}$ Theoretical Division, Los Alamos National Laboratory, Los Alamos, New Mexico 87545, USA \\ (Received 7 December 2015; revised manuscript received 24 March 2016; published 17 May 2016)

\begin{abstract}
We present a study of the collisional relaxation of ion velocities in a strongly coupled, ultracold neutral
\end{abstract} \\ plasma on short time scales compared to the inverse collision rate. The measured average velocity of a \\ tagged population of ions is shown to be equivalent to the ion-velocity autocorrelation function. We thus \\ gain access to fundamental aspects of the single-particle dynamics in strongly coupled plasmas and to the \\ ion self-diffusion constant under conditions where experimental measurements have been lacking. \\ Nonexponential decay towards equilibrium of the average velocity heralds non-Markovian dynamics \\ that are not predicted by traditional descriptions of weakly coupled plasmas. This demonstrates the \\ utility of ultracold neutral plasmas for studying the effects of strong coupling on collisional processes, \\ which is of interest for dense laboratory and astrophysical plasmas.
}

DOI: 10.1103/PhysRevX.6.021021

\section{INTRODUCTION}

In strongly coupled plasmas [1], the Coulomb interaction energy between neighboring particles exceeds the kinetic energy, leading to nonbinary collisions that display temporal correlations between past and future collision events. Such non-Markovian dynamics invalidates traditional theory for collision rates [2-4] and transport coefficients $[5,6]$ used for weakly coupled plasmas and frustrates the formulation of a tractable kinetic theory. This challenging fundamental problem is also one of the major limitations to our ability to accurately model equilibration, transport, and equations of state of dense laboratory and astrophysical plasmas [7,8], which impacts the design of inertialconfinement-fusion experiments $[9,10]$, stellar chronometry based on white dwarf stars [11,12], and models of planet formation [13]. Molecular dynamics (MD) simulations have been the principal recourse for obtaining a microscopic understanding of short-time collision dynamics in this regime [14-18], but direct comparison of results with experiment has not been possible.

In the experiments described here, we study the effects of strong coupling on collisional processes by measuring the velocity autocorrelation function (VAF) for charges in a strongly coupled plasma. The VAF, a central quantity in the statistical physics of many-body systems, encodes the influence of correlated collision dynamics and system memory on individual particle trajectories [19], and it is defined as

Published by the American Physical Society under the terms of the Creative Commons Attribution 3.0 License. Further distribution of this work must maintain attribution to the author(s) and the published article's title, journal citation, and DOI.
Subject Areas: Atomic and Molecular Physics, Plasma Physics, Statistical Physics

$$
Z(t)=\frac{1}{3}\left\langle\mathbf{v}_{k}(t) \cdot \mathbf{v}_{k}(0)\right\rangle
$$

Here, $\mathbf{v}_{k}$ is the velocity of particle $k$, and brackets indicate an equilibrium, canonical-ensemble average. Remarkably, we obtain this individual-particle quantity from measurement of the bulk relaxation of the average velocity of a tagged subpopulation of particles in an equilibrium plasma. This contrasts with measurements of macroscopic-particle VAFs based on statistical sampling of individual trajectories, which is commonly used in studies of dusty-plasma kinetics [20,21] and Brownian motion [22-24].

The VAF also provides information on transport processes since its time integral yields the self-diffusion coefficient through the Green-Kubo relation

$$
D=\int_{0}^{\infty} Z(t) d t
$$

which describes the long-time mean-square displacement of a given particle through $D=\lim _{t \rightarrow \infty}\left\langle|\mathbf{r}(t)-\mathbf{r}(0)|^{2}\right\rangle / 6 t$ [25]. Our results provide the first experimental measurement of the VAF and of self-diffusion in a threedimensional strongly coupled plasma. These results are found to be consistent with MD simulations to within the experimental uncertainty $[5,6]$.

Measurements are performed on ions in an ultracold neutral plasma (UCNP), which is formed by photoionizing a laser-cooled atomic gas [26,27]. Shortly after plasma creation, ions equilibrate in the strongly coupled regime with Coulomb coupling parameter,

$$
\Gamma_{i}=\frac{e^{2}}{4 \pi \varepsilon_{0} k_{B} T_{i}}\left(\frac{4 \pi n}{3}\right)^{1 / 3},
$$


as large as $\sim 4$. Here, $T_{i}$ is the ion temperature and $n$ is the density. Electrons in the plasma provide a neutralizing background and static screening on the ionic time scale with a Debye screening length $\lambda$. This makes UCNPs a nearly ideal realization of a Yukawa one-component plasma (OCP) [28], a paradigm model of plasma and statistical physics in which particles interact through a pairwise Coulomb potential screened by a factor $\exp (-r / \lambda)$. Ultracold plasmas are quiescent, near local equilibrium, and "clean" in the sense they are composed of a single ion species and free of strong background fields. Strong coupling is obtained at relatively low density, which slows the dynamics and makes short-time-scale processes (compared to the inverse collision rate) experimentally accessible.

Powerful diagnostics exist for dense laboratory plasmas. However, their interpretation is complicated by the transient and often nonequilibrium nature of the plasmas, and they do not provide model-independent information on the effects of particle correlations at short time scales. These include, for example, measurement of the dynamic structure factor with x-ray Thomson scattering [29-31] and measurement of electrical conductivity using a variety of techniques [29,32-35]. Comprehensive studies of selfdiffusivity [20,21,36-38] exist for strongly coupled dusty plasmas, but these systems are typically two dimensional and, therefore, do not directly illuminate the kinetics of bulk, three-dimensional plasmas.

\section{METHODS}

We perform experiments on ultracold neutral plasmas [27], which are created by first laser cooling ${ }^{88} \mathrm{Sr}$ atoms in a magneto-optical trap [39]. Atoms are then photoionized with one photon from a narrow-band laser resonant with the principal ${ }^{1} S_{0}-{ }^{1} P_{1}$ transition at $461 \mathrm{~nm}$ and another photon from a tunable 10-ns, pulsed dye laser near $413 \mathrm{~nm}$. The electron temperature $\left(T_{e}\right)$ in the plasma is determined by the excess photon energy above the ionization threshold, which can be tuned to set $T_{e}=1-1000 \mathrm{~K}$. Ions initially have very little kinetic energy, but they possess an excess of Coulomb potential energy, and they equilibrate on a microsecond time scale to a temperature $T_{i}=0.5-2.5 \mathrm{~K}$, determined primarily by the plasma density [40,41]. The ion equilibration process is called disorder-induced heating (DIH).

Disorder-induced heating limits the ions to $\Gamma_{i} \approx 2-4$. To obtain measurements on more weakly coupled systems $\left(\Gamma_{i}<1\right)$, the plasma is heated with ion acoustic waves $[42,43]$. Waves are excited by placing a grating (10 cycles/ $\mathrm{mm}$ ) in the path of the ionization beam, which is then imaged onto the magneto-optical trap for greatest contrast to create a plasma with a striped density modulation. After sufficiently long time, the waves completely damp, heating the plasma and reducing $\Gamma_{i}$. Electrons provide a uniform screening background for the ions, with screening parameter $\kappa \equiv a / \lambda=0.1-0.58$ in these experiments, for Wigner-Seitz radius $a=(3 / 4 \pi n)^{1 / 3}$.

The plasma density distribution is Gaussian in shape, $n=n_{0} \exp \left[-r^{2} / 2 \sigma(t)\right]$, with initial size $\sigma(0)=1-2 \mathrm{~mm}$. Because of electron pressure forces, the plasma expands according to $\sigma^{2}(t)=\sigma^{2}(0)\left(1+t^{2} / \tau_{\exp }^{2}\right)$, where $\tau_{\exp }=10-50 \mu \mathrm{s}$ is the expansion time [44].

An optical pump-probe technique $[4,45]$ is used to measure $\left\langle\Delta v_{x}(t)\right\rangle_{+} \equiv\left\langle v_{x}(t)\right\rangle_{+}-\left\langle v_{x}(t)\right\rangle_{\text {total }}$, the average velocity of a "spin-tagged" subpopulation of ions (labeled "+") relative to the local bulk velocity of all the ions $\left(\left\langle v_{x}(t)\right\rangle_{\text {total }}\right)$. Appendix A provides a proof that the normalized $\operatorname{VAF} \Psi(t) \equiv Z(t) / Z(0)$ is equivalent to the observable $\left\langle\Delta v_{x}(t)\right\rangle_{+} /\left\langle\Delta v_{x}(0)\right\rangle_{+}$as long as the total system is near thermal equilibrium and if terms beyond second order are negligible in a Hermite-Gauss expansion of the initial $x$-velocity distribution function for the subgroup $f_{x,+}$. As shown below, our experiment satisfies these conditions, which provides a new technique for measuring the VAF.

The evolution of $\left\langle\Delta v_{x}(t)\right\rangle_{+}$is measured by first using optical pumping to create electron-spin-tagged ion subpopulations with nonzero average velocity (Fig. 1). Pumping is accomplished by two counterpropagating, circularly polarized laser beams each detuned by the same small amount, $-\Delta_{p}$, from the ${ }^{2} S_{1 / 2}-{ }^{2} P_{1 / 2}$ transition at $421.7 \mathrm{~nm}$. Taking advantage of the unpaired electron in the ${ }^{2} S_{1 / 2}$ ground state, ions are pumped out of the $+1 / 2$ spin

(a)

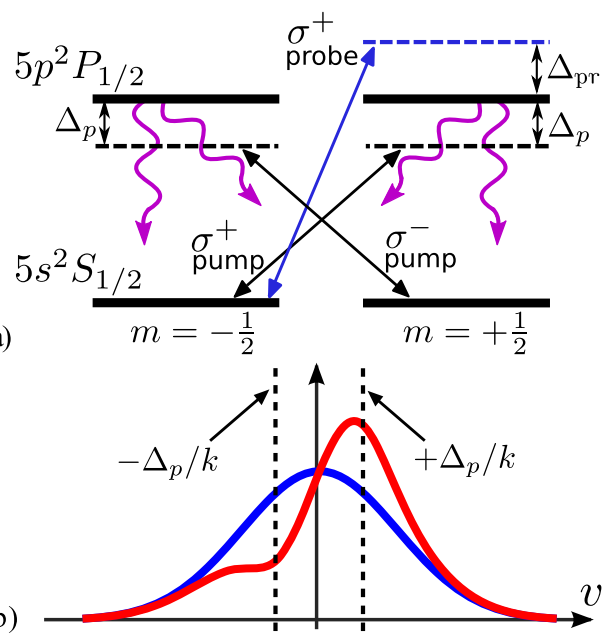

FIG. 1. (a) Optical pumping and LIF spectroscopy. Ions are optically pumped from the $+1 / 2$ to $-1 / 2$ electronic spin state around $v_{x}=-\Delta_{p} / k$, and from the $-1 / 2$ to $+1 / 2$ spin state around $v_{x}=+\Delta_{p} / k$ with two counterpropagating, circularly polarized laser beams detuned by $-\Delta_{p} \mathrm{rad} / \mathrm{s}$ from the ${ }^{2} S_{1 / 2}-{ }^{2} P_{1 / 2}$ transition of the strontium ion $(421.7 \mathrm{~nm})$. The velocity profiles of the individual spin populations are measured with LIF using a tunable circularly polarized probe beam of variable detuning $\Delta_{\mathrm{pr}}$. (b) Idealized illustration of a pumped velocity distribution for $+1 / 2$ ions resulting from optical pumping (red curve), along with an unperturbed Gaussian thermal distribution (blue curve). 
state and into the $-1 / 2$ state around the negative $x$ velocity $v_{x}=-\Delta_{p} / k$, while ions are pumped from $-1 / 2$ to $+1 / 2$ spin around the positive velocity $+\Delta_{p} / k$ (the quantization axis is taken to be along the axis of the pump beams, defined as $\hat{\mathbf{x}}$ ). This creates subpopulations of $+1 / 2$ and $-1 / 2$ spin ions having velocity distributions skewed in opposite directions, while the entire plasma itself remains in equilibrium. The pump detuning, $\Delta_{p} / 2 \pi=30 \mathrm{MHz}$, is resonant for ions with $\left|v_{x}\right|=12.6 \mathrm{~m} / \mathrm{s}$, which is on the order of the thermal velocity, $v_{\text {th }}=\sqrt{k_{B} T_{i} / M_{i}}=$ $13.7 \mathrm{~m} / \mathrm{s}$ for $T_{i}=2 \mathrm{~K}$.

We probe the ion distribution with spatially resolved laser-induced fluorescence (LIF) spectroscopy [44,46] (Fig. 1). A LIF probe beam tuned near the ${ }^{2} S_{1 / 2}-{ }^{2} P_{1 / 2}$ transition of the ${ }^{88} \mathrm{Sr}$ ion propagates along the $x$ axis and excites fluorescence that is imaged onto an intensified CCD camera with $1 \times$ magnification $(12.5 \mu \mathrm{m}$ per pixel), from which the plasma density and $x$-velocity distribution are extracted. By using a circularly polarized LIF probe beam, propagating nearly along the pump beam axis, we selectively probe only the $+1 / 2$ ions.

Pumping is applied several plasma periods $\left(2 \pi / \omega_{p}\right)$ after ionization to allow the plasma to approach equilibrium after the disorder-induced heating phase [27,40]. $\left(\omega_{p}=\sqrt{n e^{2} / \epsilon_{0} M_{i}} \sim 10^{7} \mathrm{~s}^{-1}\right.$ is the ion plasma oscillation frequency.) Electro-optic-modulator pulse pickers are used to achieve 10-ns time resolution for application of the pump and probe beams. The optical pumping time is $200 \mathrm{~ns}$, and the pump intensity is $200 \mathrm{~mW} / \mathrm{cm}^{2}$ (saturation parameter $s_{0}=3.5$ ). LIF data are taken at least $35 \mathrm{~ns}$ after the turn-off of the pump to avoid contamination of the signal with light from the decay of atoms promoted to the ${ }^{2} P_{1 / 2}$ state during the pumping process. The origin of time is taken as the middle of the 235-ns preparation time. The pumping and imaging transition is not closed, and about $1 / 15$ of the excitations result in an ion decaying to a metastable ${ }^{2} D_{3 / 2}$ state that no longer interacts with the lasers. To ensure that Larmor precession of the prepared atomic states does not contaminate the data, a 4.5-G magnetic field is applied along the pump-probe beam axis.

The LIF spectra (Fig. 2) $S(\nu)$ are fit to a convolution of a Lorentzian function $L(\nu)=\gamma_{L} /\left\{\pi\left[\left(\gamma_{L}\right)^{2}+2(\nu)^{2}\right]\right\}$ of frequency $\nu(\mathrm{Hz})$ with the one-dimensional ion-velocity distribution along the laser axis, $f_{x,+}\left(v_{x}\right)$,

$$
S(\nu)=\int_{-\infty}^{+\infty}(1 / \lambda) L(\nu-s / \lambda) f_{x,+}(s) d s
$$

where $\lambda$ is the laser wavelength and $\gamma_{L}$ is the width of Lorentzian spectral broadening. For this system, $\gamma_{L}=\gamma_{l}+\gamma_{n} \sqrt{1+s_{0}}$, where $\gamma_{l}$ is the laser linewidth (5.5 MHz) and $\gamma_{n}$ is the natural linewidth $(20.2 \mathrm{MHz})$. The width is power broadened by the laser saturation parameter $s_{0}$. The distribution $f_{x,+}$ is modeled with a Hermite-Gauss expansion,
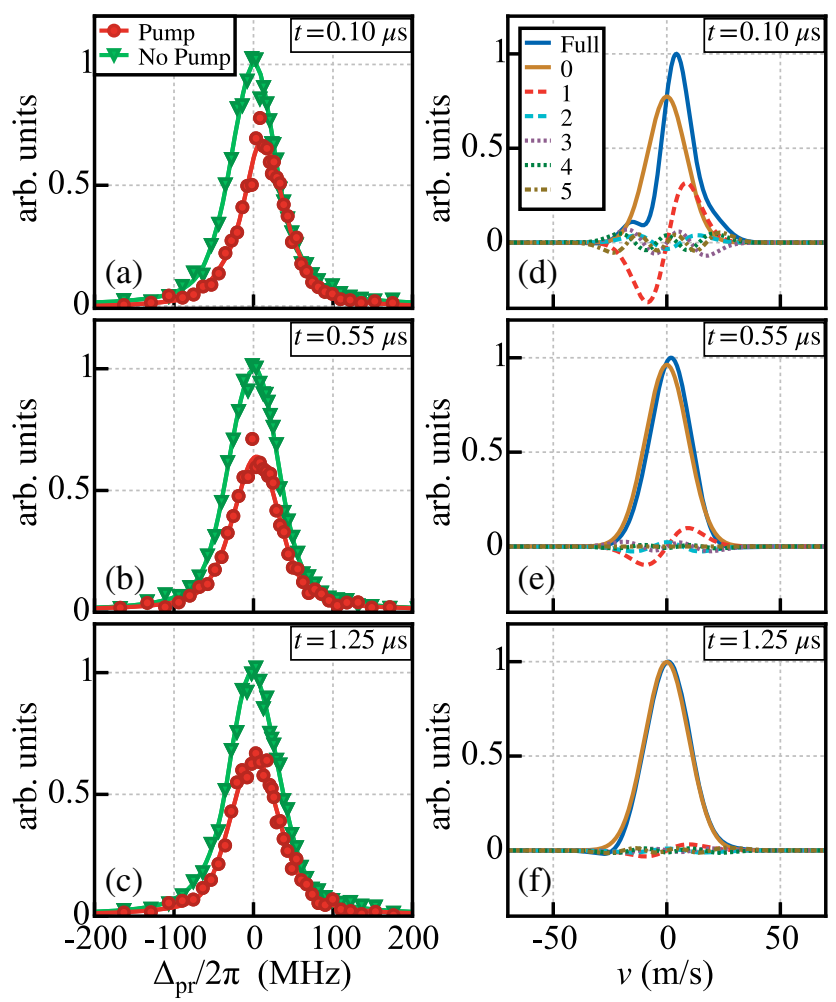

FIG. 2. (a)-(c) Time evolution of ${ }^{2} S_{1 / 2}-{ }^{2} P_{1 / 2}$ LIF spectra, pumped (red circles) and unpumped (green squares), for spin $+1 / 2$ ions in a plasma with $\bar{\kappa}=0.55$ and $\bar{\Gamma}_{i}=3.0$. (Bars denote time-averaged values; see Appendix B.) Spectra are fit (red and green lines) to a fifth-order Hermite-Gauss expansion of the velocity distribution convolved with the Lorentzian contribution from the laser and natural linewidths. (d)-(f) Full velocity distributions determined from the fits of pumped data to Eqs. (3) and (4), along with constituent Hermite terms $n=0-5$. Most of the velocity distribution is contained in the first few terms. Terms of order larger than zero decay in time as the distribution relaxes to a Maxwellian.

$$
\begin{aligned}
f_{x,+}\left(v_{x}\right)= & \frac{1}{\sigma_{v} \sqrt{2 \pi}} \exp \left\{-\frac{\left[v_{x}-\left\langle v_{x}(t)\right\rangle_{\text {total }}\right]^{2}}{2 \sigma_{v}^{2}}\right\} \\
& \times \sum_{n=0}^{N} \frac{C_{n}}{\left(2^{n} n ! \sqrt{\pi}\right)^{1 / 2}} H_{n}\left[\frac{v_{x}-\left\langle v_{x}(t)\right\rangle_{\text {total }}}{\sigma_{v}}\right],
\end{aligned}
$$

where $\sigma_{v}=\sqrt{k_{B} T_{i} / M_{i}}, H_{n}$ are Hermite polynomials, and the parameters $C_{n}$ are expansion coefficients to be determined by a fit to the data. For the analysis, $N=5$ is chosen because the amplitudes of orders 4 and higher are consistent with random noise.

The bulk velocity of the plasma $\left\langle v_{x}(t)\right\rangle_{\text {total }}$ arises because of the plasma expansion. To separate expansion from velocity perturbation due to optical pumping and thermal-velocity spread, the plasma is divided into regions that are analyzed independently [46]. $T_{i}$ and $\left\langle v_{x}(t)\right\rangle_{\text {total }}$ for each region are determined from analysis of LIF data from an unpumped plasma. LIF data from an identical plasma 


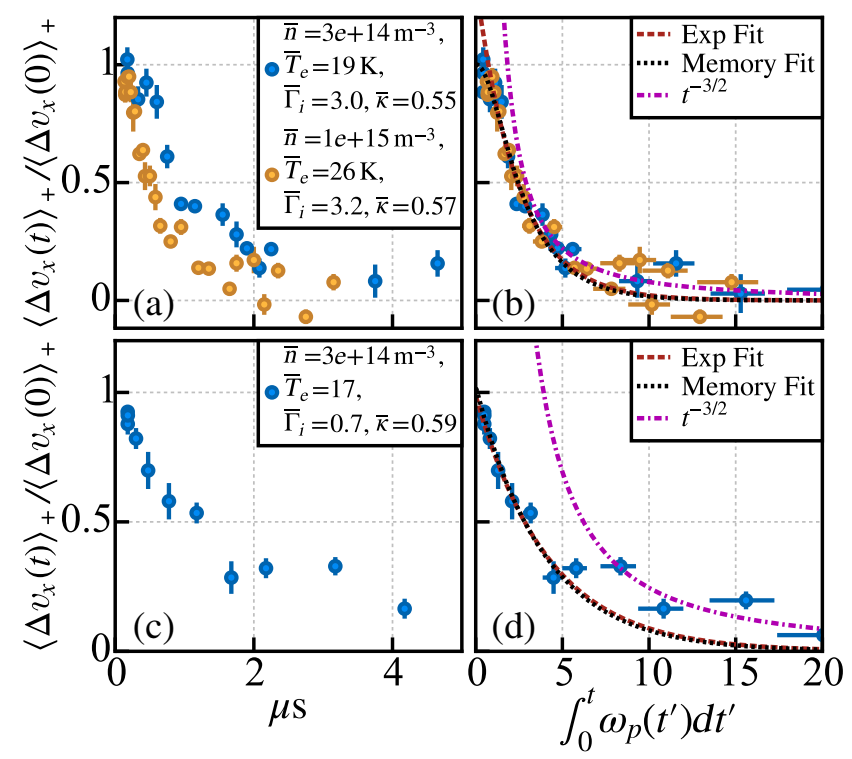

FIG. 3. Relaxation of the average velocity of spin $+1 / 2$ ions in an optically pumped plasma. Lines indicate memory-function and exponential fits to early-time data, as well as a $t^{-3 / 2}$ fit to the late-time tail. (a) $\left\langle\Delta v_{x}(t)\right\rangle_{+} /\left\langle\Delta v_{x}(0)\right\rangle_{+}$plotted versus time for two strongly coupled plasmas. (b) Data from (a) versus time scaled to the time integral of $\omega_{p}$. This plot shows the universal scaling of $\left\langle\Delta v_{x}(t)\right\rangle_{+} /\left\langle\Delta v_{x}(0)\right\rangle_{+}$with $\omega_{p}$ for plasmas of different densities but with approximately the same $\bar{\Gamma}_{i}$ and $\bar{\kappa}$. Panels (c) and (d) are the same plots as (a) and (b) but for a more weakly coupled plasma.

except with optical pumping are then fit to Eqs. (3) and (4) with only the expansion coefficients $C_{n}$ as fit parameters in order to determine $\left\langle v_{x}(t)\right\rangle_{+}$, the average velocity for + ions including expansion and the effects of pumping. From the pumped and unpumped data, we find the difference $\left\langle\Delta v_{x}(t)\right\rangle_{+}$for each of the regions, which are averaged together to arrive at final values, such as in Figs. 3 and 4. A detailed example of this analysis is provided in Appendix C.

Because of the plasma expansion, the density and thus the plasma frequency $\omega_{p}$ decrease with time. To account for this, in Figs. 3(b), 3(d), and 4 we show the time evolution of the averaged velocity as a function of the scaled time $t_{s}=\int_{0}^{t} \omega_{p}\left(t^{\prime}\right) \mathrm{d} t^{\prime}$, where the evolution of $\omega_{p}(t)$ is described assuming self-similar expansion of the Gaussian distribution [44]. All quoted plasma parameters indicated by a symbol with a bar above it, such as $\bar{\kappa}$ and $\bar{\Gamma}_{i}$, are time averages over the period of the measurement, as described in Appendix B. The density typically varies by a factor of 2 during the measurement. The normalization factor $\left\langle\Delta v_{x}(0)\right\rangle_{+}$is determined from the fit of the data using a memory-function formalism described below.

\section{RESULTS AND DISCUSSION}

Sample LIF spectra at various times after optical pumping are shown in Figs. 2(a)-2(c) for a plasma with $\bar{\kappa}=0.55$

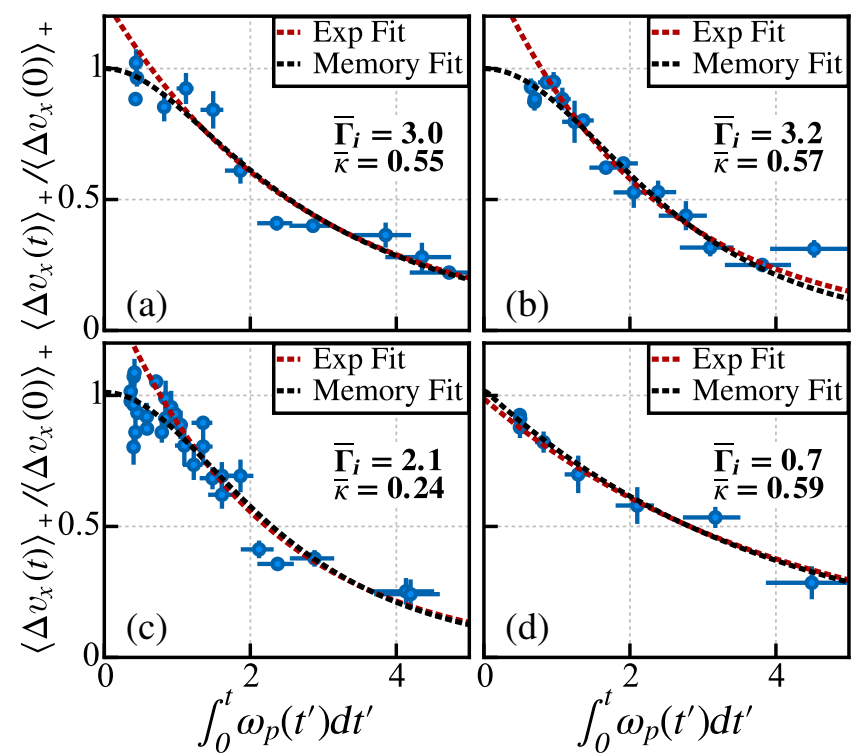

FIG. 4. Early-time behavior of $\left\langle\Delta v_{x}(t)\right\rangle_{+} /\left\langle\Delta v_{x}(0)\right\rangle_{+}$. Lines indicate memory-function and exponential fits. Data and fits for plasmas with (a) $\bar{\kappa}=0.55$ and $\bar{\Gamma}_{i}=3.0\left(\bar{T}_{e}=19 K\right)$, (b) $\bar{\kappa}=0.57$ and $\quad \bar{\Gamma}_{i}=3.2 \quad\left(\bar{T}_{e}=26 K\right), \quad(\mathrm{c}) \quad \bar{\kappa}=0.24 \quad$ and $\quad \bar{\Gamma}_{i}=2.1$ $\left(\bar{T}_{e}=88 K\right)$, and (d) $\bar{\kappa}=0.59$ and $\bar{\Gamma}_{i}=0.7 \quad\left(\bar{T}_{e}=15 K\right)$. Deviation from exponential decay is evident in more strongly coupled plasmas.

and $\bar{\Gamma}_{i}=3.0$. Figures 2(d)-2(f) show the corresponding ion-velocity distributions and individual Hermite-Gauss components of the pumped velocity distributions extracted from fits to the raw spectra. At early times, there is significant amplitude in the $n=1$ term, corresponding to the skew in the velocity distribution. This decays away as $f_{x,+}$ approaches a Maxwellian centered around $\left\langle v_{x}(t)\right\rangle_{\text {total }}$. Higher-order terms are small at all times, satisfying an important condition for the proof that $\Psi(t) \approx\left\langle\Delta v_{x}(t)\right\rangle_{+} /\left\langle\Delta v_{x}(0)\right\rangle_{+}$.

In the frame comoving with $\left\langle v_{x}(t)\right\rangle_{\text {total }}$ in a given region, the average velocity of $+1 / 2$ ions a time $t$ after optical pumping is $\left\langle\Delta v_{x}(t)\right\rangle_{+}=\int f_{x,+}\left(v_{x}\right)\left(v_{x}-\left\langle v_{x}\right\rangle_{\text {total }}\right) d v_{x} /$ $\int f_{x,+}\left(v_{x}\right) d v_{x}$. Figures 3(a) and 3(b) show sample data for two plasmas of different densities but with nearly the same values of $\kappa$ and $\Gamma_{i}$ (one with $\bar{\kappa}=0.55$ and $\bar{\Gamma}_{i}=3.0$, the other with $\bar{\kappa}=0.57$ and $\bar{\Gamma}_{i}=3.2$ ). In Fig. 3(a), data are plotted versus time, while Fig. 3(b) plots $\left\langle\Delta v_{x}(t)\right\rangle_{+} /\left\langle\Delta v_{x}(0)\right\rangle_{+}$versus time scaled by $\omega_{p}^{-1}$, appropriately accounting for the time-changing value of $\omega_{p}$. The correspondence between the two data sets apparent in Fig. 3(b) demonstrates the existence of a universal time scale for the dynamics [28]. Corresponding data for $\bar{\kappa}=0.59, \bar{\Gamma}_{i}=0.7$ are shown in Figs. 3(c) and 3(d).

\section{A. Observation of non-Markovian dynamics}

Data for $\left\langle\Delta v_{x}(t)\right\rangle_{+} /\left\langle\Delta v_{x}(0)\right\rangle_{+}$show nonexponential decay of the average velocity up to times given by 
$\int_{0}^{t} \omega_{p} d t^{\prime} \sim 1$, which is a hallmark of non-Markovian dynamics reflecting the strong coupling of the ions. This is most clearly shown in Fig. 4, which is an expanded view of the early-time data from velocity relaxation curves. The early-time behavior of $\Psi(t) \approx\left\langle\Delta v_{x}(t)\right\rangle_{+} /\left\langle\Delta v_{x}(0)\right\rangle_{+}$can be described using a memory-function formalism that treats the effects of collisional correlations at the microscopic level $[15,19,47,48]$. It can be derived from a generalized Langevin equation describing the motion of a single test particle experiencing memory effects and fluctuating forces, which is familiar from treatments of Brownian motion $[19,47]$. The evolution of the VAF is found to be

$$
\dot{Z}(t)=-\int_{0}^{t} K\left(t-t^{\prime}\right) Z\left(t^{\prime}\right) d t^{\prime} .
$$

Here, $K\left(t-t^{\prime}\right)$ is the memory function describing the influence at time $t$ from the state of the system at $t^{\prime}$.

A general, closed-form expression for $K\left(t-t^{\prime}\right)$ is lacking, but there are expressions derived from simplifying assumptions that agree well with molecular dynamics simulations for simple fluids $[19,49]$ and Yukawa potentials when $\Gamma_{i}>20[14,50]$. Some formulas introduce a time constant $\tau$ that may be interpreted as the correlation time for fluctuating forces $[15,19,47]$. If one assumes that collisions are isolated instantaneous events, $\tau \rightarrow 0$ and the memory function becomes a delta function. This is the Markovian limit in which the evolution of a system is entirely determined by its present state and $\Psi(t)$ has purely exponential dependence. Data from a more weakly coupled sample [Fig. 4(d), $\bar{\Gamma}_{i}=0.7$ ] show no discernible rollover in $\Psi(t)$ at short times.

An often-used approximation for $K\left(t-t^{\prime}\right)$ for the nonMarkovian regime, valid for short times and moderately strong coupling, is the Gaussian memory function $[15,19,47]$,

$$
K_{G}\left(t-t^{\prime}\right)=\frac{2 \gamma_{c}}{\sqrt{2 \pi \tau^{2}}} \exp \left[-\frac{\left(t-t^{\prime}\right)^{2}}{2 \tau^{2}}\right],
$$

which satisfies the conditions that memory effects vanish at long time and that it agrees with a Taylor expansion of $K\left(t-t^{\prime}\right)$ to second order around $t=t^{\prime}$ relating $\tau$ to frequency moments of the Fourier transform of the VAF [19]. For the Yukawa OCP, MD simulations have shown that Eq. (6) accurately reproduces the ion VAF for $\Gamma_{i}<10$ and $\omega_{p} t<\pi$, and the parameter $\gamma_{c}$ can be related to a well-defined collision rate [4].

Figures 3 and 4 show fits of the data in the scaled time range $0<\int_{0}^{t} \omega_{p} d t^{\prime}<4$ to Eq. (5) with a Gaussian memory function, along with exponential fits of data with $0.8<\int_{0}^{t} \omega_{p} d t^{\prime}<4.5$. At early times $\left(\int_{0}^{t} \omega_{p} d t^{\prime}<0.5\right)$, the memory-kernel fit captures the rollover, which is indicative of non-Markovian collisional dynamics. The values of $\tau$ we extract from the fit are on the order predicted by MD simulations of a classic OCP [15], although improved experimental accuracy is required before a precise comparison can be made.

\section{B. Ion VAF and self-diffusion coefficients}

Using $\left\langle\Delta v_{x}(t)\right\rangle_{+} /\left\langle\Delta v_{x}(0)\right\rangle_{+}$as an approximation for the normalized ion VAF, the self-diffusion coefficient $D$ may be calculated from our measurements. As is normally the case with calculations of this type, proper treatment of the upper limit of integration in the Green-Kubo formula is critical for obtaining accurate results. The behavior of the long-time tail of the VAF for a Yukawa system has not been explored in detail for the regime of our experiment, and this is an important area for future study. For concreteness, we assume a $t^{-3 / 2}$ dependence, which is well established as $t \rightarrow \infty$ for neutral simple fluids [19] and is generally accepted as the slowest possible decay [51]. We fit the last few data points [beyond the time $t_{\text {cut }}$, where $\int_{0}^{t_{\text {cut }}} \omega_{p}\left(t^{\prime}\right) d t^{\prime}=3 \pi$ ] to a $b t_{s}^{-3 / 2}$ curve, where $b$ is the fit parameter and $t_{s}=\int_{0}^{t} \omega_{p}\left(t^{\prime}\right) d t^{\prime}$ is the scaled time. The dimensionless self-diffusion coefficient, $D^{*} \equiv D / a^{2} \omega_{p}$, is thus calculated as

$$
D^{*} \approx \frac{1}{3 \bar{\Gamma}_{i}} \int_{0}^{t_{s, N}} Z\left(t_{s}\right) d t_{s}+\frac{2 b}{3 \bar{\Gamma}_{i}} t_{s, N}^{-1 / 2},
$$

where the first term is calculated numerically from the data by linear interpolation and the trapezoidal rule. The time of the last data point is $t_{N}$, and $t_{s, N}=\int_{0}^{t_{N}} \omega_{p}\left(t^{\prime}\right) d t^{\prime}$ in scaled units. Extracted values for $D^{*}$, along with theoretical curves for $\kappa=0$ and $\kappa=0.6$ determined from a fit to molecular dynamics simulations $[5,6]$, are shown in Fig. 5. For comparison, Fig. 5 also shows $D^{*}$ calculated from the traditional Landau-Spitzer approach and from two Chapman-Enskog calculations. Of the latter, one uses a screened Coulomb interaction that includes electron and ion screening $[52,53]$. The other uses a hypernetted chain effective potential and a modified-Enskog correction calculated in Ref. [54] (EPT-HNC/Enskog).

The error bars for $D^{*}$ include statistical and systematic contributions we describe in Appendix B. The contribution from the unmeasured long-time tail of $\Psi(t)$ adds the dominant uncertainty. The lower error bar for $D^{*}$ assumes no contribution beyond our measured points, while the upper error bar reflects a tail contribution twice as large as our best estimate. This is conservative given that the VAF is exponential in the weakly coupled limit. There are significant experimental improvements that can be made in the measurement and important systematic effects that must be investigated. The latter are scientifically interesting in their own right, such as the time scale for the approach to equilibrium of velocity correlations after plasma creation and the effect of plasma expansion on the microscopic dynamics. Any complications caused by these systematics can be greatly reduced by performing measurements 


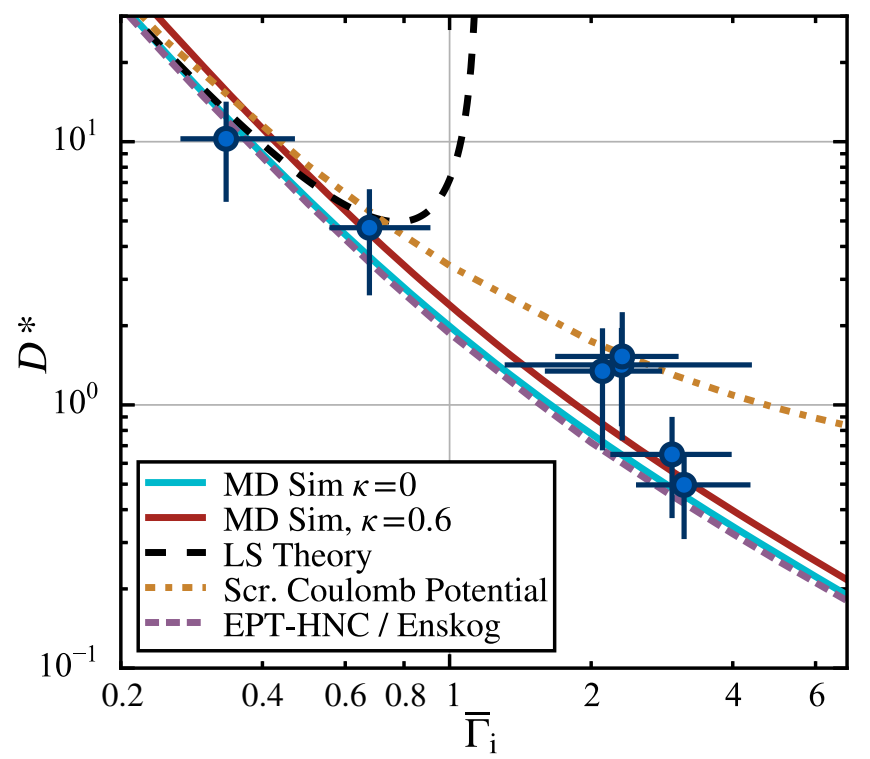

FIG. 5. Plot of the normalized self-diffusion coefficient $D^{*}$ [Eq. 7)] calculated for our data. The solid blue and solid red lines represent results from MD simulations for $\kappa=0$ and $\kappa=0.6$, respectively [5,6]. The black dashed line represents the LandauSpitzer (LS) theory for weakly coupled plasmas, which diverges in the regime of strong coupling. The orange and the purple dashed lines use screened Coulomb and hypernetted chain interactions, respectively, as described in the text.

on a larger plasma for which the expansion time scale is much greater than the characteristic collisional time scale $\left(\omega_{p} \tau_{\exp } \gg 1\right)$.

Other sources of uncertainty include variation of density across the analysis regions, the spread in bulk plasma velocity across and within regions, the time-evolving density, and the uncertainty in the density calibration. These uncertainties are reflected in the horizontal error bars in Fig. 5 and can be significantly reduced in future experiments.

\section{CONCLUSIONS}

Utilizing a spin-tagging technique to perturb and then measure the average velocity $\left\langle v_{x}(t)\right\rangle_{+}$of a subpopulation of ions in an ultracold neutral plasma, and taking advantage of an identification of the time evolution of this quantity with the normalized ion $\operatorname{VAF}[\Psi(t)]$, we experimentally measure the VAF in a strongly coupled plasma. From this, we calculate the ion self-diffusion coefficient $D$, which provides an experimental benchmark that has been lacking for molecular dynamics simulations of strongly coupled Coulomb systems in three dimensions. The data also display a nonexponential decay of $\Psi(t)$ at early times, which has not previously been observed experimentally in a bulk plasma and is indicative of non-Markovian collisional dynamics. This behavior is well described by a memoryfunction formalism.
Overall, these measurements experimentally validate foundational concepts describing how ion velocity correlations at the microscopic level determine the dynamics of strongly coupled systems at the macroscopic level, which cannot be adequately described by simple analytical methods. Because ultracold neutral plasmas offer a clean realization of the commonly used Yukawa OCP model, these results are relevant for fundamental kinetic theory and other plasmas for which effects of strong coupling are important.

\section{ACKNOWLEDGMENTS}

This work was supported by the Air Force Office of Scientific Research (FA9550-12-1-0267), Department of Energy, Fusion Energy Sciences (DE-SC0014455), and Department of Defense through the National Defense Science and Engineering Graduate Fellowship. The work of J. D. was supported by the Department of Energy Office of Fusion Energy Sciences.

\section{APPENDIX A: $\left\langle\Delta v_{x}(t)\right\rangle_{+} /\left\langle\Delta v_{x}(0)\right\rangle_{+}$AS AN APPROXIMATION TO $\Psi(t)$}

We show that the quantity $\left\langle\Delta v_{x}(t)\right\rangle_{+} /\left\langle\Delta v_{x}(0)\right\rangle_{+}$we measure in these experiments corresponds to the normalized $\operatorname{VAF}[\Psi(t)=Z(t) / Z(0)]$ if the initial velocity distribution for $+1 / 2$ ions $\left(f_{+}\right)$is Maxwellian in $v_{y}$ and $v_{z}$ and well described by a second-order Hermite-Gauss expansion in $v_{x}$, and the optical pumping prepares the subsystem of $+1 / 2$ ions in a nonequilibrium state close to thermodynamic equilibrium.

To prove this, we transform into the frame comoving with any bulk hydrodynamic velocity of the ensemble, making $\left\langle v_{x}(t)\right\rangle_{\text {total }}=0$, which does not invalidate any steps in the proof but makes $\left\langle\Delta v_{x}(t)\right\rangle_{+}=\left\langle v_{x}(t)\right\rangle_{+}$. For simplicity, we assume that the plasma is spatially homogeneous in a volume $V$, although the following arguments can be readily extended to account for nonuniform distributions. Finally, we assume that the optical pumping occurs instantaneously at some initial time $t=0$.

Let us consider a specified $+1 / 2$ ion labeled " $s$ " with position $\mathbf{r}_{s}(t)$ and velocity $\mathbf{v}_{s}(t)$ at time $t$; for a statistical description of the dynamics, it is useful to define the microscopic phase space density as $N_{s}(\mathbf{r}, \mathbf{v}, t)=$ $\delta\left(\mathbf{r}-\mathbf{r}_{s}(t)\right) \delta\left(\mathbf{v}-\mathbf{v}_{s}(t)\right)$ and its statistical average $f_{s}(\mathbf{r}, \mathbf{v}, t)$. Before optical pumping, for $t<0$, the system is in thermal equilibrium and $f_{s}(\mathbf{r}, \mathbf{v}, t)=\langle N(\mathbf{r}, \mathbf{v}, t)\rangle=$ $f_{M}(\mathbf{v}) / V$, where $f_{M}(\mathbf{v})$ is the Maxwell-Boltzmann velocity distribution. After pumping, the subsystem is out of thermal equilibrium:

$$
f_{s}(\mathbf{r}, \mathbf{v}, t)=f_{M}(\mathbf{v}) / V+\delta f_{s}(\mathbf{r}, \mathbf{v}, t) .
$$

We assume $\left|\delta f_{s} /\left(f_{M} / V\right)\right| \ll 1$, so that $\delta f_{s}$ satisfies 
$\delta f_{s}(\mathbf{r}, \mathbf{v}, t)=\int d \mathbf{r}^{\prime} d \mathbf{v}^{\prime} R_{s}\left(\mathbf{r}-\mathbf{r}^{\prime} ; \mathbf{v}, \mathbf{v}^{\prime} ; t\right) \delta f_{s}\left(\mathbf{r}^{\prime}, \mathbf{v}^{\prime}, 0\right)$,

where $R_{s}\left(\mathbf{r}-\mathbf{r}^{\prime} ; \mathbf{v}, \mathbf{v}^{\prime} ; t\right)$ is the propagator, or retarded Green's function, of the equation that governs the temporal evolution of $\delta f_{s}$, which is obtained by linearizing the exact evolution equation satisfied by $f_{s}$. Remarkably, the propagator $R_{s}$, which describes the nonequilibrium dynamics of the system, is related simply to the equilibrium time-correlation function $C_{s}\left(\mathbf{r}-\mathbf{r}^{\prime} ; \mathbf{v}, \mathbf{v}^{\prime} ; t\right)=$ $\left\langle N_{s}(\mathbf{r}, \mathbf{v}, t) N_{s}\left(\mathbf{r}^{\prime}, \mathbf{v}^{\prime}, t\right)\right\rangle$ as follows:

$$
\begin{aligned}
C_{s}\left(\mathbf{r} ; \mathbf{v}, \mathbf{v}^{\prime} ; t\right)= & \int d \mathbf{r}^{\prime} \int d \mathbf{v}^{\prime \prime} R_{s}\left(\mathbf{r}-\mathbf{r}^{\prime} ; \mathbf{v}, \mathbf{v}^{\prime \prime} ; t\right) \\
& \times C_{S}\left(\mathbf{r}^{\prime} ; \mathbf{v}^{\prime \prime}, \mathbf{v}^{\prime} ; 0\right) \\
= & R_{S}\left(\mathbf{r} ; \mathbf{v}, \mathbf{v}^{\prime} ; t\right) f_{M}\left(\mathbf{v}^{\prime}\right) / V
\end{aligned}
$$

where, in the last equality, we use the initial value $C_{s}\left(\mathbf{r} ; \mathbf{v}, \mathbf{v}^{\prime} ; 0\right)=\delta(\mathbf{r}) \delta\left(\mathbf{v}-\mathbf{v}^{\prime}\right) f_{M}(\mathbf{v}) / V$. This relation is an expression of the fluctuation-dissipation theorem [55].

From Eq. (A2), the average particle velocity along the $x$ direction determined in our experiment is

$$
\begin{aligned}
\left\langle v_{x}(t)\right\rangle_{+} & =\int d \mathbf{r} d \mathbf{v} v_{x} \delta f_{s}(\mathbf{r}, \mathbf{v}, t) \\
& =\int d \mathbf{v} \int d \mathbf{v}^{\prime} v_{x} \bar{R}_{s}\left(\mathbf{v}, \mathbf{v}^{\prime} ; t\right) \delta \bar{f}_{s}\left(\mathbf{v}^{\prime}, 0\right),
\end{aligned}
$$

where $\bar{R}_{s}\left(\mathbf{v}, \mathbf{v}^{\prime} ; t\right)=\int d \mathbf{r} R_{s}\left(\mathbf{r} ; \mathbf{v}, \mathbf{v}^{\prime} ; t\right)$ and $\delta \bar{f}_{s}(\mathbf{v}, 0)=$ $\int d \mathbf{r} \delta f_{s}(\mathbf{r}, \mathbf{v}, 0)$. If, as found experimentally (see Fig. 2), $\delta \bar{f}_{s}$ is initially well described by a second-order HermiteGauss expansion in $v_{x}$, then

$$
\begin{aligned}
\left\langle v_{x}(t)\right\rangle_{+}= & \int d \mathbf{v} \int d \mathbf{v}^{\prime} v_{x}\left[c_{0}+c_{1} v_{x}^{\prime}+c_{2}\left(v_{x}^{\prime}\right)^{2}\right] \\
& \times \bar{R}_{s}\left(\mathbf{v}, \mathbf{v}^{\prime} ; t\right) f_{M}\left(\mathbf{v}^{\prime}\right) / V \\
= & \int d \mathbf{v} \int d \mathbf{v}^{\prime} v_{x}\left[c_{0}+c_{1} v_{x}^{\prime}+c_{2}\left(v_{x}^{\prime}\right)^{2}\right] \\
& \times \bar{C}_{s}\left(\mathbf{v}, \mathbf{v}^{\prime} ; t\right),
\end{aligned}
$$

where we use the fluctuation-dissipation theorem Eq. (A3) to obtain the second equality. The zeroth- and second-order terms vanish since $\bar{C}_{s}\left(\mathbf{v}, \mathbf{v}^{\prime} ; t\right)=\bar{C}_{s}\left(-\mathbf{v},-\mathbf{v}^{\prime} ; t\right)$, and the previous result simplifies to

$$
\begin{aligned}
\left\langle v_{x}(t)\right\rangle_{+} & =c_{1} \int d \mathbf{v} \int d \mathbf{v}^{\prime} v_{x} v_{x}^{\prime} \bar{C}_{s}\left(\mathbf{v}, \mathbf{v}^{\prime} ; t\right) \\
& =c_{1}\left\langle v_{x, s}(t) v_{x, s}(0)\right\rangle=c_{1} Z(t) .
\end{aligned}
$$

Therefore, we obtain the desired result:

$$
\frac{\left\langle v_{x}(t)\right\rangle_{+}}{\left\langle v_{x}(0)\right\rangle_{+}}=\frac{Z(t)}{Z(0)}=\Psi(t)
$$

\section{APPENDIX B: VARIATION AND UNCERTAINTIES OF PLASMA PARAMETERS}

Because the plasmas in these experiments undergo expansion during the measurements, the values of various plasma parameters quoted for a given VAF measurement are time-averaged values given by a trapezoidal approximation to $\bar{\Theta}=\left[\int_{0}^{t_{f}} \Theta\left(t^{\prime}\right) d t^{\prime}\right] / t_{f}$, where $t_{f}$ is the total time of measurement and $\Theta(t)$ represents $T_{i}(t), n_{i}(t), \kappa(t), \omega_{p}(t)$, or $\Gamma_{i}(t)$.

We now discuss the effects of expansion on the values of the various plasma parameters, as well as the methods used to incorporate these systematic variations into the quoted uncertainties. UCNPs are created by ionizing neutral atoms in a cloud with a 3D Gaussian profile. Immediately after ionization, $T_{i}$ equals the temperature of the trapped atoms (around $10 \mathrm{mK}$ ), but on the time scale of the inverse plasma frequency, the ions heat to a few degrees kelvin due to disorder-induced heating. Measurements of the velocity autocorrelation function and the self-diffusion constant are performed after the DIH is complete and the plasma is near local thermal equilibrium. After DIH, the ion temperature still changes due to electron-ion heating and cooling from expansion into the vacuum [56], but this is a slow evolution compared to the experimental time scale.

After ionization, the plasma expands due to electron pressure [44]. The expansion of the plasma cloud is selfsimilar and well described by $\sigma(t)^{2}=\sigma(0)^{2}\left(1+t^{2} / \tau_{\exp }^{2}\right)$, where $\sigma(t)$ is the Gaussian width parameter at time $t$ after ionization and $\tau_{\exp }$ is the expansion time scale defined by $\tau_{\exp }=\sqrt{m_{i} \sigma(0)^{2} / k_{B}\left[T_{e}(0)+T_{i}(0)\right]}$, where $m_{i}$ is the ion mass, $k_{B}$ is Boltzmann's constant, $T_{e}(0)$ is the initial electron temperature, and $T_{i}(0)$ is the ion temperature after DIH. Because of this self-similar expansion, an infinitesimal spatial region of the plasma that starts at a given normalized radial position $\tilde{\mathbf{r}}(0)=\eta$ remains centered at $\tilde{\mathbf{r}}=\eta$ throughout the expansion, where $\tilde{\mathbf{r}}(t)=\mathbf{r}(t) / \sigma(t)$. To track a well-defined population of ions in the plasma, the analysis regions move with the plasma expansion at a fixed $\tilde{\mathbf{r}}$, while also increasing in size at the same rate as $\sigma(t)$.

The density is determined from the amplitude of the LIF spectrum [46], with initial calibration given by the DIH curve as described in Ref. [28]. The density at a position of constant $\tilde{\mathbf{r}}$ changes by as much as a factor of 2 during the course of a measurement, but $\omega_{p}$ and $\Gamma_{i}$ vary as $n^{1 / 2}$ and $n^{1 / 3}$, respectively, leading to a much smaller change in these quantities.

The gradient of velocity outward from the center of the plasma broadens the LIF spectrum in proportion to the length of the analysis region along the direction of 

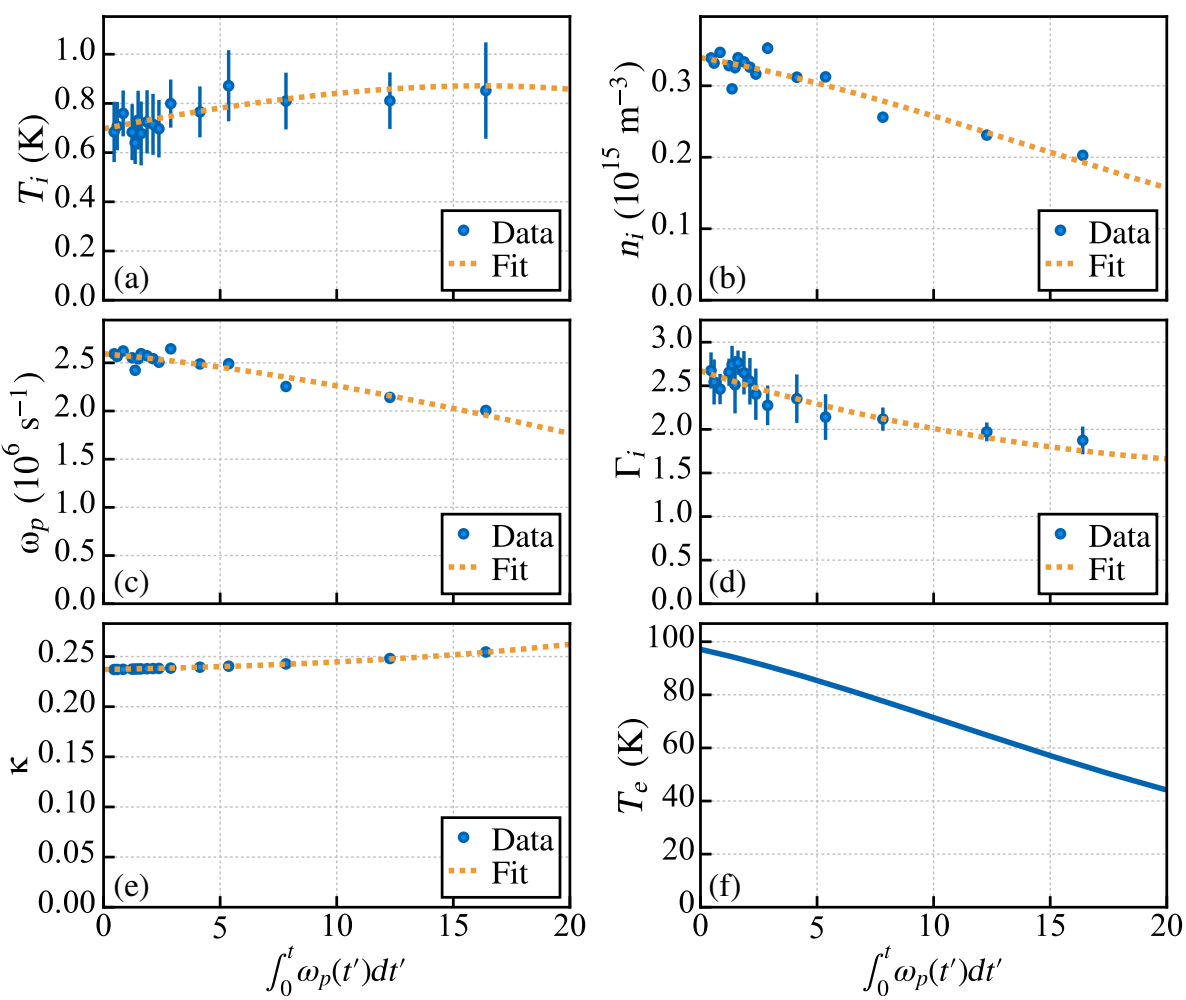

FIG. 6. Typical evolution of (a) $T_{i}$, (b) $n_{i}$, (c) $\omega_{p}$, (d)

(d) $\Gamma_{i}$, (e) $\kappa$, and (f) $T_{e}$ versus scaled time with smooth fits.

propagation of the LIF laser, which complicates the determination of plasma temperature. Small regions are used (corresponding to $50 \mu \mathrm{m}$ along the $k$ vector of the LIF beam), so that the velocity change over a region is less than $10 \%$ of the thermal velocity at the very latest times. During most of the measurement, it is significantly less.

The effects of expansion are illustrated for a plasma with $\bar{\kappa}=0.24$ and $\bar{\Gamma}_{i}=2.1$ in Fig. 6 . This plasma has one of the fastest expansions in the data set, and represents a "worst case" for the complications introduced by plasma expansion. In Fig. 6, the averages of $T_{i}$ and $n_{i}$ for all the analysis regions are shown with vertical error bars representing the standard deviation. $\omega_{p}$ and $\Gamma_{i}$ are calculated from $T_{i}$ and $n_{i}$ for each region and then averaged. Data are fit to smooth functions in time, which are used to calculate $\omega_{p}(t)$ in the expressions for scaled times in Figs. 2 and 3, and to determine the values of time-averaged $\bar{\Gamma}_{i}$ used to plot the self-diffusion coefficient data in Fig. 5. The smooth fit to $T_{i}$ is a second-order polynomial, while the fits to the remaining quantities are made using the plasma expansion formulas [44]. The initial electron temperature $T_{e}(0)$ is determined by the detuning of the photoionization laser above threshold [27], and the evolution $T_{e}(t)$ is assumed to follow predictions for a self-similar plasma expansion [44].

The spread in the value of $\Gamma_{i}$ during a single measurement of the self-diffusion constant, as shown in Fig. 6, is taken as the systematic uncertainty of $\bar{\Gamma}_{i}$ in Fig. 5, and is reflected in the error bars. Statistical uncertainties are also reflected in the error bars, but they are a minor contribution. The spread in $\omega_{p}$ values is also taken as the systematic uncertainty of scaled time $t_{s}$, which propagates into the calculation of the self-diffusion constant through the Green-Kubo integral of $\left\langle\Delta v_{x}(t)\right\rangle_{+} /\left\langle\Delta v_{x}(0)\right\rangle_{+}$over the scaled time. Overall, the estimate of the total uncertainties in $\bar{\Gamma}_{i}$ and $D^{*}$ is a conservative one, incorporating statistical uncertainty, systematic uncertainty arising from incomplete knowledge of the form of the long-time tail of the VAF, and "uncertainty" introduced by the range of values the plasma parameters take over the course of the measurement.

\section{APPENDIX C: SPECTRA FOR INDIVIDUAL REGIONS AND VARIATION ACROSS THE PLASMA}

In Fig. 7(a), spectra from individual analysis regions are shown for the plasma described in Fig. 6. The unpumped spectra exhibit a shift of the center frequency from zero that increases with time and distance from the plasma center (region 0), reflecting the plasma expansion. By fitting the unpumped spectra to a Gaussian distribution convolved with the laser and natural linewidth broadening (assumed to be a Lorentzian function), the center frequency and Gaussian width are extracted. The center frequency determines the bulk expansion velocity $\tilde{v}_{x}$ for each region. The width determines the ion temperature $T_{i} . T_{i}$ and $\tilde{v}_{x}$ are set as constants in fits of the corresponding pumped 

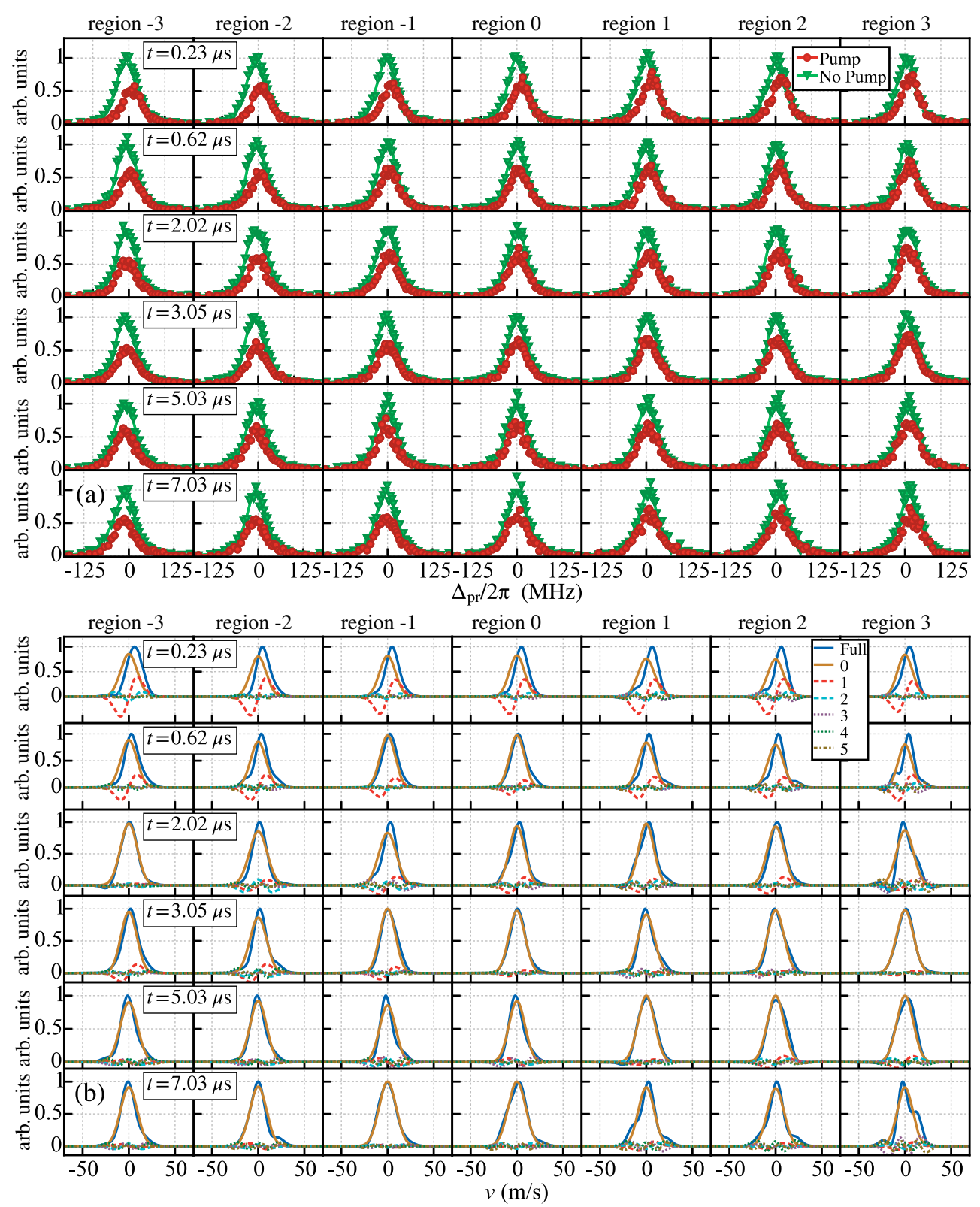

FIG. 7. (a) Pumped and unpumped LIF spectra from regions of width $0.1 \sigma_{x}$ across the plasma cloud spanning $x= \pm 0.2 \sigma_{x}$ for the plasma described in Fig. 6. (b) Pumped and unpumped velocity distributions determined from the spectra shown in (a).

distributions to the fifth-order Hermite expansion [Eq. (5)] convolved with the Lorentzian broadening. As Fig. 7(a) shows, the fit to the raw data captures the "skewing" of pumped distributions well. The convolution of the distribution with the Lorentzian broadening preserves the first moment of the distributions, so this broadening should not affect the value of the average velocity.

In Fig. 7(b), the underlying velocity distributions from the fits to each raw spectrum in Fig. 7(a) are shown, along with each term of the Hermite expansion. Together, Figs. 7(a) and 7(b) demonstrate that the first-order Hermite term dominates over the higher-order odd terms at early times across all the regions, which is a requirement for
$\left\langle\Delta v_{x}(t)\right\rangle_{+} /\left\langle\Delta v_{x}(0)\right\rangle_{+}$to be identified as the normalized velocity autocorrelation function.

To disentangle the effects of plasma expansion from thermal ion motion in the LIF spectra, it is important to use regions that are relatively small along the direction of propagation of the LIF laser beam. Seven small, overlapping regions are analyzed individually. The regions have widths $0.1 \sigma$ along the direction of propagation of the LIF laser and span from $-0.2 \sigma_{x}$ to $+0.2 \sigma_{x}$ along the same direction.

The values of $\left\langle\Delta v_{x}(t)\right\rangle_{+}$for each of the seven regions are averaged together to get one time evolution of $\left\langle\Delta v_{x}(t)\right\rangle_{+}$ for the plasma. A typical result is shown in Fig. 8 for the plasma described in Fig. 6. Black points are the average of 


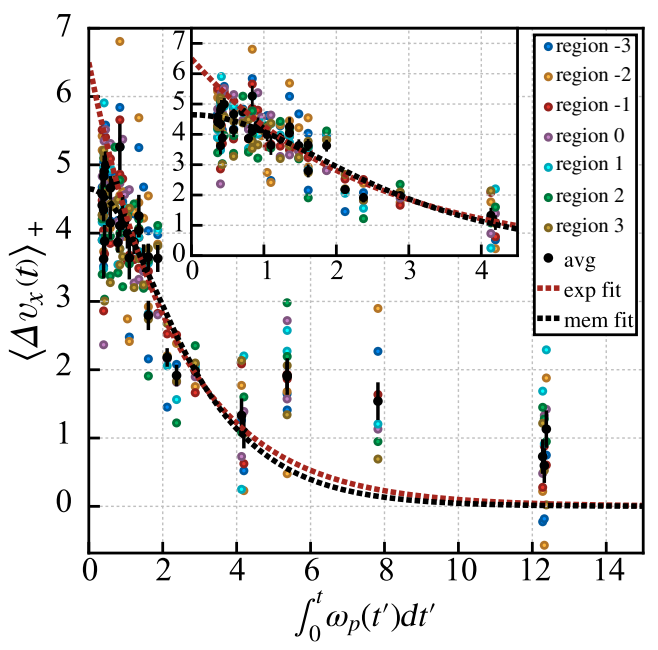

FIG. 8. Evolution of average velocity of pumped spectra across all regions for the plasma described in Fig. 6. The black circles represent the mean value for each time point. The inset represents a closeup view of the same data over a scaled time ranging from 0 to 4.5 to highlight the nonexponential decay at early times.

all regions at each time. The error bars are the statistical, one-standard-deviation uncertainties of the mean values, which we use in the main article to calculate the uncertainties in $\left\langle\Delta v_{x}(t)\right\rangle_{+} /\left\langle\Delta v_{x}(0)\right\rangle_{+}$. The fits to the exponential function (using data in the range $0.8<\omega_{p} t<4$ ) and to the memory kernel (using data in the range $0<\omega_{p} t<4$ ) are also shown for reference.

[1] S. Ichimaru, Strongly Coupled Plasmas: High-Density Classical Plasmas and Degenerate Electron Liquids, Rev. Mod. Phys. 54, 1017 (1982).

[2] L. Spitzer, Physics of Fully Ionized Gases, Interscience Tracts on Physics and Astronomy (Interscience Publishers, New York, 1962), Vol. 3.

[3] L. D. Landau, Kinetic Equation for the Coulomb Effect, Phys. Z. Sowjetunion 10, 154 (1936).

[4] G. Bannasch, J. Castro, P. McQuillen, T. Pohl, and T. C. Killian, Velocity Relaxation in a Strongly Coupled Plasma, Phys. Rev. Lett. 109, 185008 (2012).

[5] J. Daligault, Diffusion in Ionic Mixtures across Coupling Regimes, Phys. Rev. Lett. 108, 225004 (2012).

[6] J. Daligault, Practical Model for the Self-Diffusion Coefficient in Yukawa One-Component Plasmas, Phys. Rev. E 86, 047401 (2012).

[7] M. S. Murillo, Strongly Coupled Plasma Physics and High Energy-Density Matter, Phys. Plasmas 11, 2964 (2004).

[8] H. M. V. Horn, Dense Astrophysical Plasmas, Science 252, 384 (1991).

[9] J. D. Lindl, P. Amendt, R. L. Berger, S. G. Glendinning, S. H. Glenzer, S. W. Haan, R. L. Kauffman, O. L. Landen, and L. J. Suter, The Physics Basis for Ignition Using Indirect-Drive Targets on the National Ignition Facility, Phys. Plasmas 11, 339 (2004).
[10] R. P. Drake, High-Energy-Density Physics: Fundamentals, Inertial Fusion, and Experimental Astrophysics (Springer Science and Business Media, New York, 2006).

[11] J. Daligault and M. S. Murillo, Semiclassical Model for the Ionic Self-Diffusion Coefficient in White Dwarfs, Phys. Rev. E 71, 036408 (2005).

[12] E. García-Berro, S. Torres, L. G. Althaus, I. Renedo, P. Lorén-Aguilar, A. H. Córsico, R. D. Rohrmann, M. Salaris, and J. Isern, A White Dwarf Cooling Age of 8 Gyr for NGC 6791 from Physical Separation Processes., Nature (London) 465, 194 (2010).

[13] B. A. Remington, R. M. Cavallo, M. J. Edwards, D. D.-M. Ho, B. F. Lasinski, K. T. Lorenz, H. E. Lorenzana, J. M. Mcnaney, S. M. Pollaine, and R. F. Smith, Accessing High Pressure States Relevant to Core Conditions in the Giant Planets, Astrophys. Space Sci. 298, 235 (2005).

[14] H. Gould and G. F. Mazenko, Microscopic Theory of Self-Diffusion in a Classical One-Component Plasma, Phys. Rev. A 15, 1274 (1977).

[15] J. P. Hansen, I. R. McDonald, and E. L. Pollock, Statistical Mechanics of Dense Ionized Matter. III. Dynamical Properties of the Classical One-Component Plasma, Phys. Rev. A 11, 1025 (1975).

[16] S. Hamaguchi, R. T. Farouki, and D. H. E. Dubin, Triple Point of Yukawa Systems, Phys. Rev. E 56, 4671 (1997).

[17] R. E. Rudd, W. H. Cabot, K. J. Caspersen, J. A. Greenough, D. F. Richards, F. H. Streitz, and P. L. Miller, Self-Diffusivity and Interdiffusivity of Molten Aluminum-Copper Alloys under Pressure, Derived from Molecular Dynamics, Phys. Rev. E 85, 031202 (2012).

[18] H. D. Whitley, D. M. Sanchez, S. Hamel, A. A. Correa, and L. X. Benedict, Molecular Dynamics Simulations of Warm Dense Carbon, Contrib. Plasma Phys. 55, 390 (2015).

[19] U. Balucani and M. Zoppi, Dynamics of the Liquid State, Oxford Series on Neutron Scattering in Condensed Matter (Clarendon Press, Oxford, 1995), Vol. 10.

[20] W.-T. Juan and L. I, Anomalous Diffusion in Strongly Coupled Quasi-2D Dusty Plasmas, Phys. Rev. Lett. 80, 3073 (1998).

[21] S. Nunomura, D. Samsonov, S. Zhdanov, and G. Morfill, Self-Diffusion in a Liquid Complex Plasma, Phys. Rev. Lett. 96, 015003 (2006).

[22] Y. W. Kim and J.E. Matta, Long-Time Behavior of the Velocity Autocorrelation: A Measurement, Phys. Rev. Lett. 31, 208 (1973).

[23] R. Huang, I. Chavez, K. M. Taute, B. Lukic, S. Jeney, M. G. Raizen, and E.-L. Florin, Direct Observation of the Full Transition from Ballistic to Diffusive Brownian Motion in a Liquid, Nat. Phys. 7, 576 (2011).

[24] S. Kheifets, A. Simha, K. Melin, T. Li, and M. G. Raizen, Observation of Brownian Motion in Liquids at Short Times: Instantaneous Velocity and Memory Loss, Science 343, 1493 (2014).

[25] A. Einstein, On the Movement of Small Particles Suspended in a Stationary Liquid Demanded by the Molecular-Kinetic Theory of Heat, Ann. Phys. (Berlin) 17, 549 (1905).

[26] T. C. Killian, S. Kulin, S. D. Bergeson, L. A. Orozco, C. Orzel, and S. L. Rolston, Creation of an Ultracold Neutral Plasma, Phys. Rev. Lett. 83, 4776 (1999). 
[27] T. C. Killian, T. Pattard, T. Pohl, and J. M. Rost, Ultracold Neutral Plasmas, Phys. Rep. 449, 77 (2007).

[28] T. K. Langin, T. Strickler, N. Maksimovic, P. McQuillen, T. Pohl, D. Vrinceanu, and T. C. Killian, Demonstrating Universal Scaling for Dynamics of Yukawa OneComponent Plasmas after an Interaction Quench, Phys. Rev. E 93, 023201 (2016).

[29] P. Sperling, E. J. Gamboa, H. J. Lee, H. K. Chung, E. Galtier, Y. Omarbakiyeva, H. Reinholz, G. Röpke, U. Zastrau, J. Hastings, L. B. Fletcher, and S. H. Glenzer, Free-Electron X-Ray Laser Measurements of CollisionalDamped Plasmons in Isochorically Heated Warm Dense Matter, Phys. Rev. Lett. 115, 115001 (2015).

[30] A. L. Kritcher, P. Neumayer, J. Castor, T. Döppner, R. W. Falcone, O. L. Landen, H. J. Lee, R. W. Lee, E. C. Morse, A. Ng, S. Pollaine, D. Price, and S. H. Glenzer, Ultrafast X-Ray Thomson Scattering of Shock-Compressed Matter, Science 322, 69 (2008).

[31] S. H. Glenzer, O. L. Landen, P. Neumayer, R. W. Lee, K. Widmann, S. W. Pollaine, R. J. Wallace, G. Gregori, A. Höll, T. Bornath, R. Thiele, V. Schwarz, W.-D. Kraeft, and R. Redmer, Observations of Plasmons in Warm Dense Matter, Phys. Rev. Lett. 98, 065002 (2007).

[32] V. N. Korobenko, A. D. Rakhel, A. I. Savvatimski, and V. E. Fortov, Measurement of the Electrical Resistivity of Hot Aluminum Passing from the Liquid to Gaseous State at Supercritical Pressure, Phys. Rev. B 71, 014208 (2005).

[33] J. Adams, N. Shilkin, V. Fortov, V. Gryaznov, V. Mintsev, R. Redmer, H. Reinholz, and G. Röpke, Coulomb Contribution to the Direct Current Electrical Conductivity of Dense Partially Ionized Plasmas, Phys. Plasmas 14, 062303 (2007).

[34] Z. Chen, B. Holst, S. E. Kirkwood, V. Sametoglu, M. Reid, Y. Y. Tsui, V. Recoules, and A. Ng, Evolution of ac Conductivity in Nonequilibrium Warm Dense Gold, Phys. Rev. Lett. 110, 135001 (2013).

[35] R. L. Shepherd, D. R. Kania, and L. A. Jones, Measurement of the Resistivity in a Partially Degenerate, Strongly Coupled Plasma, Phys. Rev. Lett. 61, 1278 (1988).

[36] L.-J. Hou, A. Piel, and P. K. Shukla, Self-Diffusion in 2D Dusty-Plasma Liquids: Numerical-Simulation Results, Phys. Rev. Lett. 102, 085002 (2009).

[37] B. Liu and J. Goree, Superdiffusion in Two-Dimensional Yukawa Liquids, Phys. Rev. E 75, 016405 (2007).

[38] T. Ott, M. Bonitz, Z. Donkó, and P. Hartmann, Superdiffusion in Quasi-Two-Dimensional Yukawa Liquids, Phys. Rev. E 78, 026409 (2008).

[39] S. B. Nagel, C. E. Simien, S. Laha, P. Gupta, V. S. Ashoka, and T. C. Killian, Magnetic Trapping of Metastable ${ }^{3} P_{2}$ Atomic Strontium, Phys. Rev. A 67, 011401 (2003).
[40] M. S. Murillo, Using Fermi Statistics to Create Strongly Coupled Ion Plasmas in Atom Traps, Phys. Rev. Lett. 87, 115003 (2001).

[41] Y. C. Chen, C. E. Simien, S. Laha, P. Gupta, Y. N. Martinez, P. G. Mickelson, S. B. Nagel, and T. C. Killian, Electron Screening and Kinetic Energy Oscillations in a Strongly Coupled Plasma, Phys. Rev. Lett. 93, 265003 (2004).

[42] J. Castro, P. McQuillen, and T. C. Killian, Ion Acoustic Waves in Ultracold Neutral Plasmas, Phys. Rev. Lett. 105, 065004 (2010).

[43] T. C. Killian, P. McQuillen, T. M. O'Neil, and J. Castro, Creating and Studying Ion Acoustic Waves in Ultracold Neutral Plasmas, Phys. Plasmas 19, 055701 (2012).

[44] S. Laha, P. Gupta, C. E. Simien, H. Gao, J. Castro, T. Pohl, and T.C. Killian, Experimental Realization of an Exact Solution to the Vlasov Equations for an Expanding Plasma, Phys. Rev. Lett. 99, 155001 (2007).

[45] J. Castro, G. Bannasch, P. McQuillen, T. Pohl, and T. C. Killian, Creating Non-Maxwellian Velocity Distributions in Ultracold Plasmas, AIP Conf. Proc. 1421, 31 (2012).

[46] J. Castro, H. Gao, and T. C. Killian, Using Sheet Fluorescence to Probe Ion Dynamics in an Ultracold Neutral Plasma, Plasma Phys. Controlled Fusion 50, 124011 (2008).

[47] J. Hansen and I. McDonald, Theory of Simple Liquids (Elsevier Science, New York, 2006).

[48] D. Levesque and L. Verlet, Computer "Experiments" on Classical Fluids. III. Time-Dependent Self-Correlation Functions, Phys. Rev. A 2, 2514 (1970).

[49] T. Gaskell and S. Miller, Longitudinal Modes, Transverse Modes and Velocity Correlations in Liquids. I, J. Phys. C 11, 3749 (1978).

[50] T. Gaskell and O. Chiakwelu, Coupling of Single Particle and Collective Modes in a One-Component Plasma (Theory Applicable to Liquids), J. Phys. C 10, 2021 (1977).

[51] T. Haxhimali, R. E. Rudd, W. H. Cabot, and F. R. Graziani, Diffusivity in Asymmetric Yukawa Ionic Mixtures in Dense Plasmas, Phys. Rev. E 90, 023104 (2014).

[52] S. D. Baalrud and J. Daligault, Effective Potential Theory for Transport Coefficients across Coupling Regimes, Phys. Rev. Lett. 110, 235001 (2013).

[53] R. L. Liboff, Transport Coefficients Determined Using the Shielded Coulomb Potential, Phys. Fluids 2, 40 (1959).

[54] S. D. Baalrud and J. Daligault, Modified Enskog Kinetic Theory for Strongly Coupled Plasmas, Phys. Rev. E 91, 063107 (2015).

[55] J. A. Krommes, Equilibrium Statistical Constraints and the Guiding-Center Plasma, Phys. Fluids B 5, 650 (1993).

[56] P. McQuillen, T. Strickler, T. Langin, and T. C. Killian, Ion Temperature Evolution in an Ultracold Neutral Plasma, Phys. Plasmas 22, 033513 (2015). 